\title{
P-Cable High-Resolution Seismic
}

By Sverre Planke, Frode Norman Eriksen, Christian Berndt, Jürgen Mienert, and Doug Masson

P-Cable technology was developed to acquire detailed three-dimensional images of the subsurface in a cost-efficient way. The system was improved and used in the HERMES project to image cold seep sites and slide areas in the Gulf of Cádiz and the Mediterranean and Barents seas.

\section{THE CONCEPT}

The P-Cable system consists of 12 to 24 very short streamers that are towed on a cross-cable that runs perpendicular to the ship's steaming direction. This configuration allows a number of seismic profiles to be acquired simultaneously in a cost-efficient way. The current $\mathrm{P}$-Cable record is 24 simultaneously collected lines. A three-dimensional subsurface image can be obtained by collecting a number of parallel sail lines in the region of interest.

Conventional three-dimensional seismic technology relies on very long streamers (commonly, several streamers up to10-km long), large sources, and costly operations. In contrast, the P-Cable system is lightweight and can be deployed quickly from small vessels. Only a small source is required as the system is optimized for relatively shallow imaging, typically down to subbottom depths similar to the water depth.

The P-Cable system is particularly useful for acquisition of small threedimensional cubes of $10-50 \mathrm{~km}^{2}$ in focus areas, rather than extensive mapping of large regions. Rapid deployment and recovery of the system makes it possible to acquire several three-dimensional cubes during one research cruise.

\section{HERMES}

Volcanic Basin Petroleum Research (VBPR) participated in the HERMES project as a small-to-medium enterprise (SME; i.e., fewer than 50 employees). VBPR's main objective was to further develop the patented P-Cable technology and use it during research cruises to image subsurface structures of relevance to the HERMES project. The project was performed in close collaboration with National Oceanography Centre, Southampton (NOCS) and the University of Tromsø.

Two cruises were completed during the project. The first went to the Håkon Mosby mud volcano in the Barents Sea with R/V Jan Mayen in 2005, and one three-dimensional cube was acquired. The second cruise went to the Gulf of Cádiz and western Mediterranean with RRS Charles Darwin in 2006; four three-dimensional cubes were acquired in both mud volcano and mudslide provinces.

The P-Cable1 system belonging to NOCS was used during both cruises. For VBPR, the cruise results provided important documentation about the usefulness of P-Cable technology. For instance, seismic data from the Gulf of Cádiz clearly documents that the P-Cable concept works very well even in deep-water areas.

\section{THE ROAD AHEAD}

The HERMES cruises inspired new ideas for possible improvements of the technology. A second-generation, digital P-Cable system - the P-Cable2-was subsequently developed during 2006 and 2007 in collaboration with the University of Tromsø, NOCS, and IFM-GEOMAR and industry partners. The P-Cable2 system was later used with success during four R/V Jan Mayen cruises offshore Norway in 2007 and 2008.

A new company, P-Cable 3D Seismic AS, was established in 2008 to further develop and commercialize P-Cable technology. Although limited, HERMES funding provided opportunities for testing and development of a new technology that will likely have important applications for marine research in academia and industry in the years to come.

\section{ACKNOWLEDGEMENTS}

We thank the crews and scientific parties of R/V Jan Mayen and R/V Charles Darwin, funded by HERMES (EU contract GOCE-CT-20055112341) and by the Norwegian Research Council (EUROMARGIN project 158733/V30; and PETROMAKS 169514/S30 and 175969/S30). ש]

\section{Sverre Planke (planke@vbpr.no) is CEO, Volcanic Basin Petroleum} Research, Oslo, Norway. Frode Norman Erikson is CMO/Geologist, Volcanic Basin Petroleum Research, Oslo, Norway. Christian Berndt is Professor, IFM-GEOMAR, Kiel, Germany. Jürgen Mienert is Professor, University of Tromsø, Tromsø, Norway. Doug Masson is Senior Researcher, National Oceanography Centre, University of Southampton, Southampton, UK.

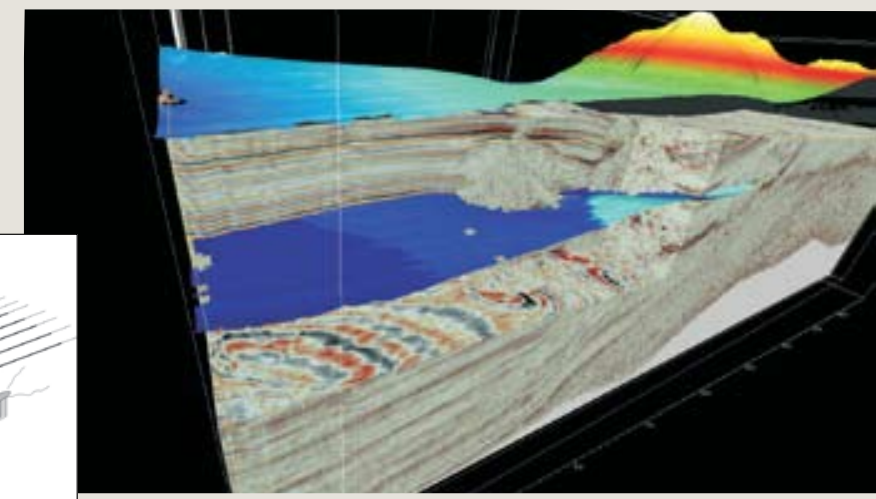

Figure 2. Example of three-dimensional seismic data acquired from the Mercator mud volcano in the Gulf of Cádiz. 